
on the acquisition of spatial memory in rats with CAl, but not large hippocampal lesions: a possible role for schema formation

\author{
Amber C. Ocampo, ${ }^{1}$ Larry R. Squire, ${ }^{2,3}$ and Robert E. Clark $^{2,4}$ \\ ${ }^{1}$ Department of Psychology, University of California, San Diego, La Jolla, California 92093, USA; ${ }^{2}$ Veterans Affairs San Diego Healthcare \\ System, San Diego, California 92161, USA; ${ }^{3}$ Departments of Psychiatry, Neurosciences, and Psychology, University of California, San \\ Diego, La Jolla, California 92093, USA; ${ }^{4}$ Department of Psychiatry, University of California, San Diego, La Jolla, California 92093, USA
}

\begin{abstract}
Prior experience has been shown to improve learning in both humans and animals, but it is unclear what aspects of recent experience are necessary to produce beneficial effects. Here, we examined the capacity of rats with complete hippocampal lesions, restricted CAl lesions, or sham surgeries to benefit from prior experience. Animals were tested in two different spatial tasks in the watermaze, the conventional watermaze task and delayed match-to-position. The two lesions impaired performance in both tasks when rats had no prior experience. However, when given prior training with one task, CAl lesions had no effect on performance in the other task. In contrast, rats with hippocampal lesions did not benefit from prior training. The findings show that prior experience can benefit learning even when the previously learned task and a new task are quite different. The concept of schema may be useful for understanding the benefits of prior experience.
\end{abstract}

Schemas refer to preexisting knowledge structures into which newly acquired information can be incorporated (Bartlett 1932; Tse et al. 2007; Dragoi and Tonegawa 2013; McKenzie et al. 2014). A variety of studies have demonstrated that preexisting knowledge (or schemas) is advantageous for human learning (Bransford \& Johnson 1972; Maguire et al. 1999; van Kesteren et al. 2013; Race et al. 2015). Although the schema concept is fundamental to the psychological science of human memory, the concept has only recently become relevant in work with experimental animals.

A number of studies have now documented striking effects of prior experience on learning in rodents. For example, in one notable study, rats learned to associate six specific flavors with six places in a familiar arena (Tse et al. 2007). Initial learning was slow, but after rats had accumulated experience in the task, they learned new flavor-place pairings in a single trial. Prior experience can also benefit learning in the watermaze task (Bannerman et al. 1995). Specifically, D-AP5 normally impairs watermaze acquisition in rats, presumably by blocking the induction of LTP, but this treatment had no effect on acquisition when rats received prior watermaze training with a different platform location in a different environment. The same benefit of prior experience has been reported using other methods to block LTP (Otnaess et al. 1999; Inglis et al. 2013) and with other tasks (Wiltgen et al. 2011; Dragoi and Tonegawa 2013). Interestingly, the benefit of prior watermaze training was not obtained in rats with conventional hippocampal lesions (Bannerman et al. 1995).

It is unclear to what extent the beneficial effects of prior experience depend on the kind of experience that an animal brings to a new task. Can beneficial effects occur when the task to be learned is

\section{Corresponding author: Isquire@ucsd.edu}

Article is online at http://www.learnmem.org/cgi/doi/10.1101//m.046482. 117. different than the task that has provided prior experience? In the current study, rats with two kinds of hippocampal lesions and control rats were given experience with the delayed match-to-position task (DMP) (Steele and Morris 1999) before training on the watermaze. Separate groups of rats were given experience with the watermaze task before training on the DMP task (Figure 1). The DMP task is similar to the watermaze task but requires rats to learn a new platform location each day. Thus, the DMP task does not provide any specific spatial information that would be useful in the conventional watermaze task (and vice versa). Training in each task does, however, provide substantial experience in a circular pool of water and experience with features common to the two tasks (e.g., a platform is to be found, the platform is not located near the walls, and distal spatial cues are important).

We tested rats with conventional hippocampal lesions and control rats. Because beneficial effects of prior experience have not been found after conventional lesions, we also tested animals with a recently developed, novel lesion restricted to field CA1 that encompasses the entire dorsoventral extent of the hippocampus (Ocampo et al. 2017). Area CA1 serves as the primary output pathway from the hippocampus to neocortex (van Strien et al. 2009). Accordingly, a complete CA1 lesion should block hippocampal output to neocortex but leave the majority of the hippocampus intact. In this way, we evaluated the effects of a more limited disruption of hippocampal function. The CA1 lesion might be advantageous because it spares efferent projections from CA3

(C) 2018 Ocampo et al. This article is distributed exclusively by Cold Spring Harbor Laboratory Press for the first 12 months after the full-issue publication date (see http://learnmem.cshlp.org/site/misc/terms.xhtml). After 12 months, it is available under a Creative Commons License (AttributionNonCommercial 4.0 International), as described at http://creativecommons. org/licenses/by-nc/4.0/. 
and/or because it reduces remote effects to structures that project to the hippocampus. Hippocampal lesions have been reported to cause volume loss in the cortex (Jarrard and Meldrum 1993; Anagnostaras et al. 2001).

\section{RESULTS}

\section{Neurohistological findings}

In the CA1 groups, all animals sustained significant damage to both the dorsal and ventral regions of area CA1. Figure $2 \mathrm{~A}$ shows two sections from a sham animal, and Figure $2 \mathrm{~B}$ shows the extent of a representative CA1 lesion. The mean percent damage to area CA1 was $76.1 \%$. Sparing occurred most frequently in the posteriormost extent of CA1. There was also typically some extra-CA1 damage in area CA3 $(24.3 \%)$ and the DG (12.6\%). Additionally, there was some damage to structures immediately adjacent to area CA1, which included the normal and unavoidable cortical damage often observed above the dorsal hippocampus. In three rats, there was also mild damage in the posteromedial, amygdalohippocampal, and amygdalopiriform transition areas near ventral CA1. However, this damage did not seem to affect performance as these three rats performed similarly to the other rats in their respective groups.

In the $\mathrm{H}$ groups, all animals sustained significant damage to both the dorsal and ventral regions of the hippocampus. Figure 2C depicts the extent of a representative hippocampal lesion. The mean percent damage to the total hippocampus was $75.2 \%$. The damage was most complete in areas CA1 (85.8\%) and CA3 $(86.9 \%)$. Sparing occurred most frequently in the dorsal- and ventral-most extents of the DG, although DG damage was still substantial (57.6\%). Sparing also often occurred in the posterior-most extent of the hippocampus. Additionally, there was some damage to structures immediately adjacent to the hippocampus, which included cortical damage above the dorsal hippocampus. In two rats, there was also mild damage to the primary and secondary auditory cortices near the intermediate hippocampus, and in one of these two rats this damage extended to the temporal association, ectorhinal, and perirhinal cortices. Again, this extra damage did not seem to affect performance as these two rats performed similarly to the other rats in their respective groups.

Figure 2D shows the mean percent damage in each hippocampal subregion for the CA1 groups and for the H groups. There was $62.6 \%$ more CA3 damage and $45.0 \%$ more DG damage in the $\mathrm{H}$
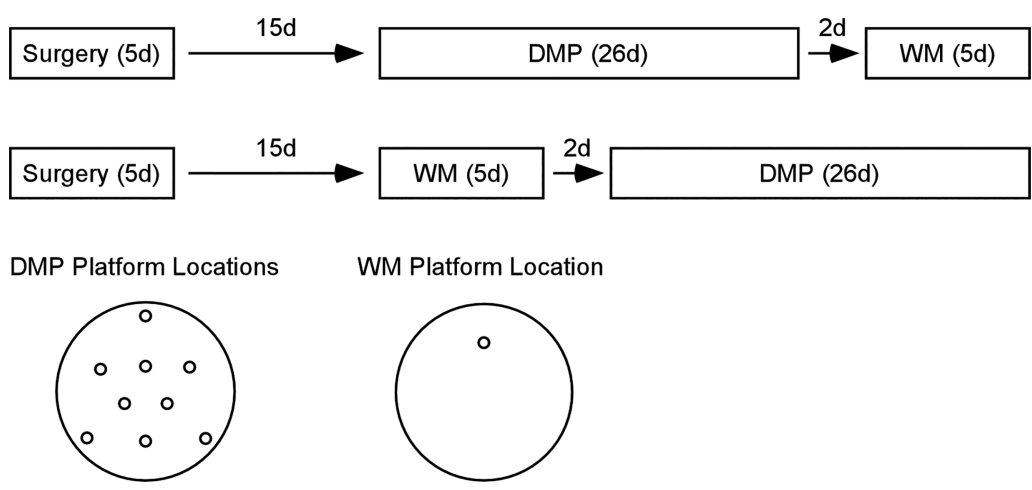

Figure 1. Experimental design. Approximately $15 \mathrm{~d}$ after receiving $\mathrm{H}$ lesions, $\mathrm{CA} 1$ lesions, or sham surgeries, rats were trained in the delayed match-to-position (DMP) task, followed $2 \mathrm{~d}$ later by the watermaze (WM) task (top timeline). Different animals with the same lesions were trained on the WM task first, followed $2 \mathrm{~d}$ later by the DMP task (bottom timeline). The diagram at the bottom left shows the different platform locations used for the DMP task (where a new platform location was used each day). The diagram at the bottom right shows the single platform location used for the WM task. group compared to the CA1 group (CA3: $t_{(46)}=27.4, P<0.0001$; DG: $\left.t_{(46)}=10.4, P<0.0001\right)$. There was also $9.7 \%$ more CA1 damage in the $\mathrm{H}$ group compared to the CA1 group $\left(t_{(46)}=3.1, P<0.005\right)$.

\section{Behavioral findings}

\section{Watermaze acquisition}

In the WM First condition, where rats had no prior training, a repeated-measures ANOVA revealed differences in acquisition rate between the $\mathrm{H}, \mathrm{CA} 1$, and control groups $\left(F_{(2,21)}=7.1, P<\right.$ 0.005) (Fig. 3A, left). Post hoc, pair-wise comparisons using the Tukey-Kramer test $(\alpha=0.05)$ showed that the two lesion groups learned the platform location at a similar rate, and both groups were impaired relative to controls. A repeated-measures ANOVA also demonstrated differences between the three groups when DMP training was given prior to the watermaze task (DMP First condition; $\left.F_{(2,20)}=6.5, P<0.01\right)$ (Fig. $3 \mathrm{~A}$, right). However, in contrast to the findings for the WM First condition, in the DMP First condition the CA1 group acquired the watermaze at the same rate as controls. The $\mathrm{H}$ group was impaired relative to the other two groups (Tukey-Kramer test, $\alpha=0.05$ ).

Together, these results indicate that prior DMP training substantially improved watermaze acquisition, but only in rats with CA1 lesions. A repeated-measures ANOVA showed that the CA1 group in the DMP First condition outperformed the CA1 group in the WM First condition $\left(F_{(1,14)}=15.4, P<0.005\right)$. A similar but smaller benefit of prior DMP training appeared for the $\mathrm{H}$ and sham groups, but the effects were marginal $\left(F_{(1,14)}=3.6, P=0.08\right.$ for the $\mathrm{H}$ group; $F_{(1,13)}=3.8, P=0.07$ for the sham group).

\section{DMP performance}

In the DMP First condition, where rats had no prior training, a one-way ANOVA revealed differences in performance between the $\mathrm{H}, \mathrm{CA} 1$, and control groups during phase one on the first day of DMP training $\left(F_{(2,20)}=7.1, P<0.005\right)$ (Fig. 4 A, left). Post hoc, pairwise comparisons showed that the two lesion groups were impaired relative to controls (Tukey-Kramer test, $\alpha=0.05$ ), and that the two lesion groups performed similarly to each other. A one-way ANOVA also demonstrated differences in performance on the first day of DMP training between the three groups when watermaze training was given prior to the DMP task $\left(F_{(2,21)}=6.8\right.$, $P<0.01)$ (Fig. $4 \mathrm{~A}$, right). However, in contrast to the findings for the DMP First condition, in the WM First condition the CA1 group performed as well as controls. The $\mathrm{H}$ group was impaired relative to the other two groups (Tukey-Kramer test, $\alpha=0.05$ ).

These results for rats with CA1 lesions indicate that prior watermaze training improved initial DMP performance. That is, on the first day of training on the DMP task, the CA1 group in the WM First condition outperformed the CA1 group in the DMP First condition $\left(t_{(14)}=5.2, P<0.0005\right)$. A similar benefit of prior watermaze training was also observed for the $\mathrm{H}$ and sham groups (all ts $>3.4$, all $P s<0.005$ ), although the $\mathrm{H}$ group remained impaired. This benefit of watermaze training on DMP performance may have resulted from rats learning and remembering certain features of the task structure during their previous experience in the watermaze (i.e., searching for a platform to escape the water, 

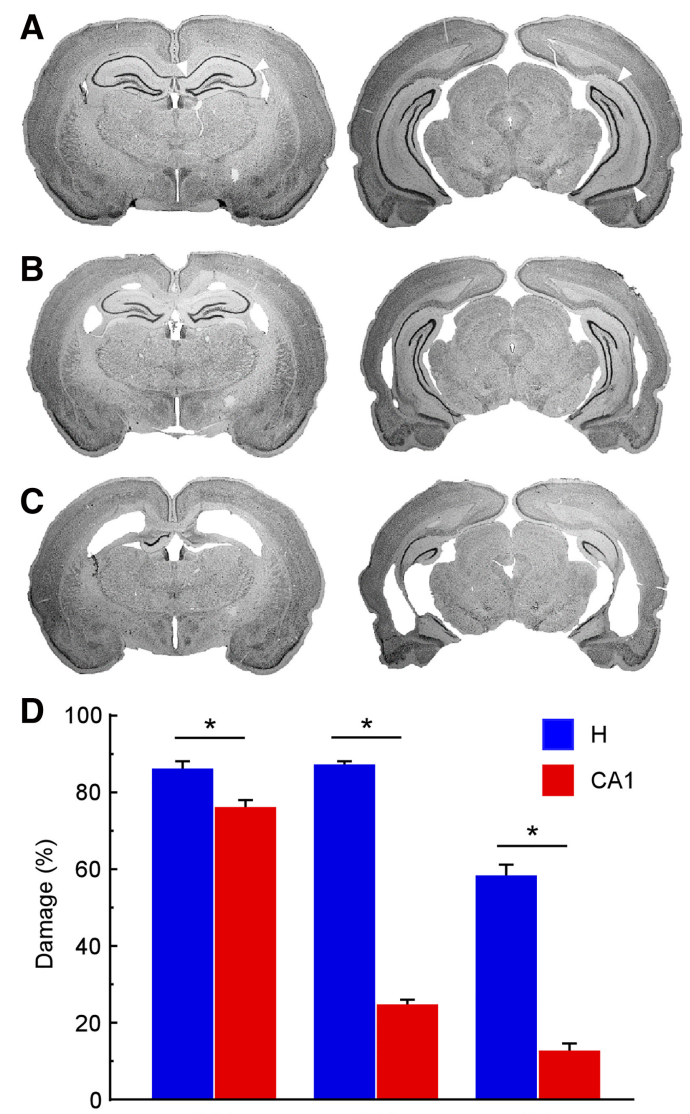

CA1

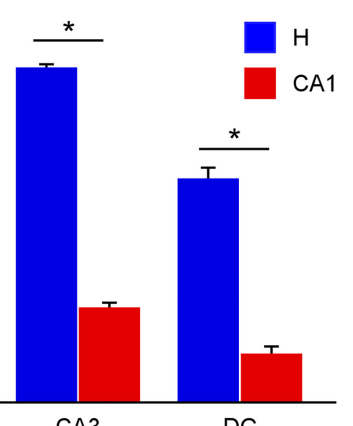

Figure 2. CA1 lesions were selective to area $\mathrm{CA} 1$, while hippocampal lesions encompassed the majority of the hippocampus. Both lesions included the entire dorsoventral extent of the hippocampus. Photomicrographs at two coronal levels of a representative $(A)$ sham brain, $(B)$ a brain with a CA1 lesion, and $(C)$ a brain with a hippocampal lesion. (Left to right) The sections are -2.80 and $-5.40 \mathrm{~mm}$ posterior to bregma. White arrows indicate the CA1 borders in each section for the sham animal. (D) Mean percent damage to area CA1, area CA3, and the DG for animals with CA1 lesions $(n=24)$ and hippocampal lesions $(n=24)$. $\left({ }^{*}\right)$ denotes significant group difference, $P<0.005$. Error bars indicate SEM.

swimming in the middle of the pool instead of along the edges, and using distal spatial cues as navigational guides).

During the remaining $4 \mathrm{~d}$ of phase one testing, the CA1 group was no longer impaired, regardless of prior experience (data not in Fig. 4). In the DMP First condition, mean distance swum across days $2-5$ was $3.3 \pm 0.5,7.1 \pm 1.0$, and $1.8 \pm 0.3 \mathrm{~m}$ for the CA1, $\mathrm{H}$, and sham groups, respectively. A repeated-measures ANOVA demonstrated differences in performance between the three groups $\left(F_{(2,20)}=16.2, P<0.0001\right)$. Post hoc, pairwise comparisons showed that the CA1 group performed similarly to controls, and the $\mathrm{H}$ group was impaired relative to the other two groups (TukeyKramer test, $\alpha=0.05)$. The results were the same in the WM First condition $(1.7 \pm 0.2,6.0 \pm 0.8,1.4 \pm 0.07 \mathrm{~m}$ for the $\mathrm{CA} 1, \mathrm{H}$, and sham groups, respectively; $F_{(2,21)}=25.9, \quad P<0.0001$; TukeyKramer test, $\alpha=0.05$ ).

Similar findings were obtained during phase two testing. In the DMP First condition (Fig. 4B, left), a repeated-measures ANOVA revealed differences in performance between the three groups across the three delays $\left(F_{(2,20)}=17.8, P<0.0001\right)$. Post hoc, pairwise comparisons showed that the CA1 group performed as well as controls, and the $\mathrm{H}$ group was impaired relative to the other two groups (Tukey-Kramer test, $\alpha=0.05$ ). The same findings were obtained when watermaze training was given prior to the DMP task $\left(F_{(2,21)}=18.3, P<0.0001\right.$; Tukey-Kramer test, $\left.\alpha=0.05\right)$ (Fig. 4B, right). There were no main effects of delay (DMP First: $F_{(2,20)}=1.4, P>0.1 ;$ WM First: $\left.F_{(2,21)}=0.5, P>0.1\right)$.

During phase three testing, CA1 lesions also did not impair performance. In the DMP First condition (Fig. 4C, left), a one-way ANOVA demonstrated differences between the three groups in the percent time spent in the platform location during the probe trial $\left(F_{(2,20)}=9.7, P<0.005\right)$. Post hoc, pairwise comparisons showed that the CA1 group performed similarly to controls $(P=0.19)$, and the $\mathrm{H}$ group was impaired relative to the other two groups (Tukey-Kramer test, $\alpha=0.05$ ). The results were the same in the WM First condition $\left(F_{(2,21)}=9.2, P<0.005\right.$; Tukey-Kramer test, $\alpha$ $=0.05)$ (Fig. 4C, right).

\section{Watermaze 43 d post surgery}

In the watermaze task, rats with CA1 lesions performed much better when they were given prior DMP training than when they had no prior training (Fig. 3A, right). In this group, the interval between surgery and watermaze training was $43 \mathrm{~d}$. By comparison, the surgery-watermaze interval for the CA1 group not given prior DMP training was only $15 \mathrm{~d}$ (Fig. 3A, left; timelines in Fig. 1). To determine whether improved performance in the CA1 group was related to the extended surgery-watermaze training interval (43 vs. $15 \mathrm{~d}$ ), we assessed the performance of rats with $\mathrm{H}$ lesions or CA1 lesions on the watermaze task $43 \mathrm{~d}$ after surgery and without prior DMP training (timeline in Fig. 5).

Watermaze acquisition was impaired in both lesion groups 43 $\mathrm{d}$ after surgery. A repeated-measures ANOVA showed that the $\mathrm{H}$

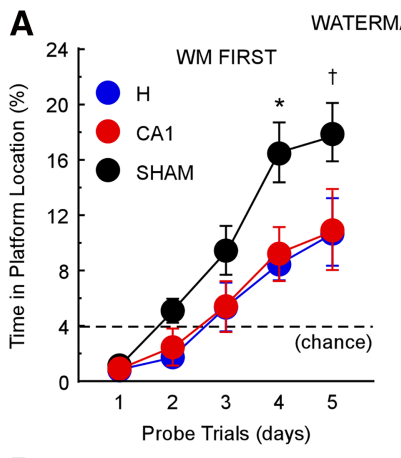

B
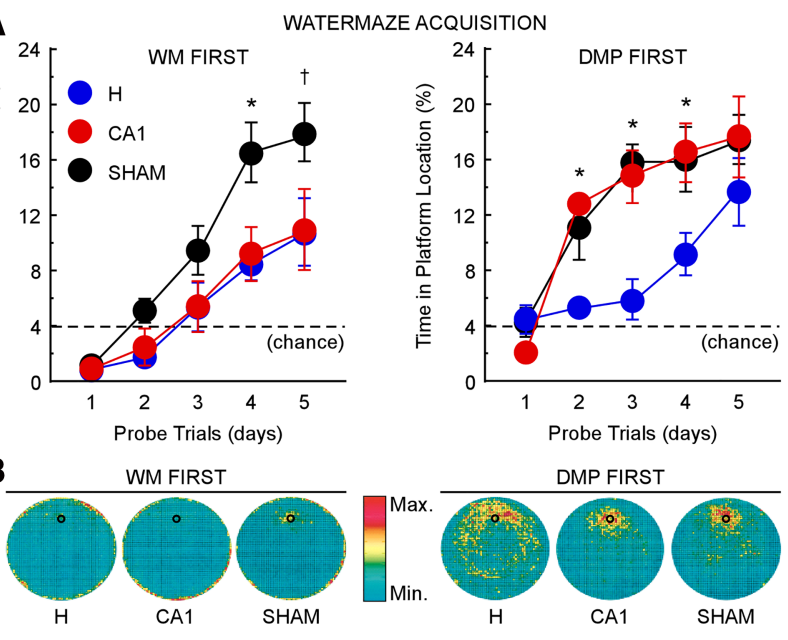

Figure 3. $C A 1$ lesions impaired $W M$ acquisition similarly to $H$ lesions when rats had no prior experience (WM First, left). However, the lesions had no effect on WM acquisition when rats previously had DMP training (DMP First, right). $\mathrm{H}$ lesions impaired WM acquisition regardless of prior training. (A) Performance of $\mathrm{H}$ (WM First $n=8$; DMP First $n=8$ ), CA1 (WM First $n=8$; DMP First $n=8$ ), and sham (WM First $n=8$; DMP First $n=7$ ) groups across $5 \mathrm{~d}$ of WM training, measured as the mean percent time spent in the platform location during a probe trial at the beginning of each training day. The dashed line indicates chance performance $(4 \%)$. (B) Heat maps represent the time spent in different parts of the watermaze on probe trials during acquisition by the two $\mathrm{H}$ groups, the two CA1 groups, and the two sham groups. For the color scale, red corresponds to the most frequently visited areas and turquoise to the least visited areas. Small black circles indicate platform location; error bars indicate SEM; $\left(^{*}\right) P<0.05$ between one group and the other two groups; ( $\dagger$ ) $P<0.05$ between sham and $\mathrm{H}$ and $P<0.07$ between sham and CA1. 
group was slightly more impaired than the H group in the WM First condition, where the watermaze task was given only $15 \mathrm{~d}$ after surgery $\left(F_{(1,14)}=6.9, P<0.05\right)$ (Fig. $5 \mathrm{~A}$, left). The CA1 group was impaired similarly to the CA1 group in the WM First condition $\left(F_{(1,14)}=0.7, P>0.1\right)$ (Fig. $5 \mathrm{~A}$, right). Together these findings indicate that the extended surgery-training interval was not the cause of the good watermaze performance of CA1 rats that received prior DMP training (Fig. 3A, right).
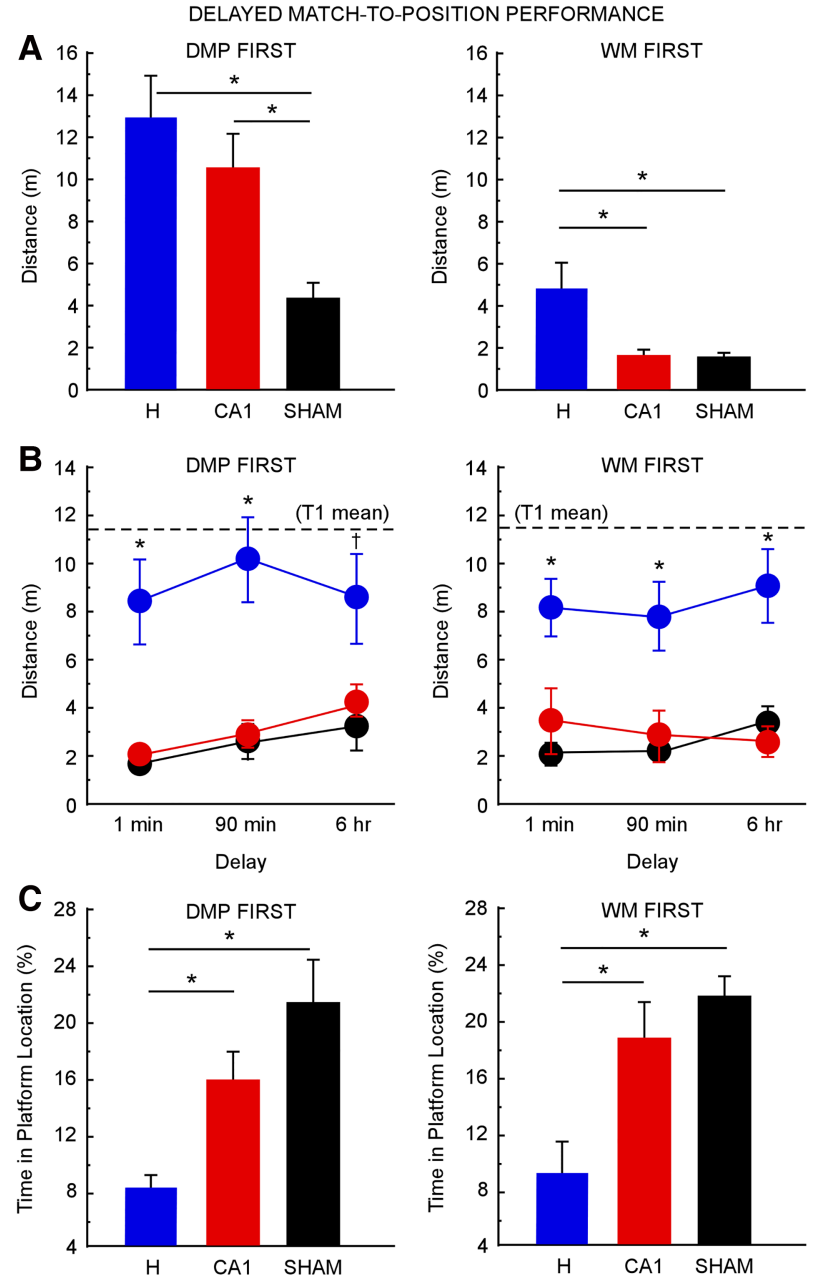

Figure 4. DMP performance after $\mathrm{H}$ lesions (DMP First $n=8$; WM First $n=8$ ), after CA1 lesions (DMP First $n=8$; WM First $n=8$ ), and for sham animals (DMP First $n=7$; WM First $n=8$ ). CA1 lesions impaired early DMP performance when rats had no prior experience (DMP First, left), but the lesions had no effect when rats had prior WM training (WM First, right). $\mathrm{H}$ lesions impaired DMP performance regardless of prior training. (A) Performance on the first day of DMP testing during phase one (four trials separated by 15 -sec delays), measured as the mean distance traveled to reach the platform on each trial. $\left(^{*}\right) P<0.05$. (B) Performance during the second phase of DMP testing (two trials separated by 1-min, 90-min, or 6-h delays) across the three delays, measured as the mean distance traveled to reach the platform during trial 2. Dashed lines indicate the distance traveled to reach the platform in trial 1 (T1) averaged across all three groups at all three delays (DMP First $=11.4 \mathrm{~m}$; WM First $=11.5 \mathrm{~m}$; the three groups in each condition performed similarly in $\mathrm{T} 1) ;\left({ }^{*}\right) P<0.05$ between the $\mathrm{H}$ group and the other two groups; ( $\dagger$ ) $P<0.05$ between $\mathrm{H}$ and sham and $P<0.06$ between $\mathrm{H}$ and CA1. (C) Performance during the last phase of DMP testing (one trial and one test probe separated by a 1-min delay), measured as the mean percent time spent in the platform location during the test probe. Chance performance was $4 \%$. $\left(^{*}\right) P<0.05$. Error bars indicate SEM.
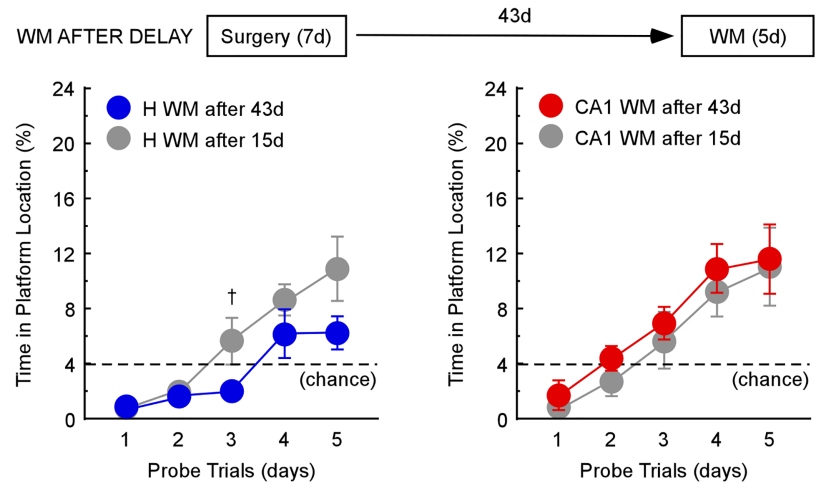

Figure 5. Rats with $\mathrm{H}$ lesions or $\mathrm{CA} 1$ lesions were trained on the WM task $43 \mathrm{~d}$ after surgery (and without prior DMP training). WM acquisition was impaired after $\mathrm{H}$ lesions (blue, left; $n=8$ ) as well as after CA1 lesions (red, right; $n=8$ ). For comparison, WM data are included in each panel from Figure 3 (left) for the WM First condition (gray; $\mathrm{H} n=8$; CA1 $n=8$ ), where animals were given the WM task $15 \mathrm{~d}$ after surgery (also without prior DMP training). Note that controls given the watermaze task without prior DMP training attained scores above $16 \%$ by day 4 (Figure 3 , left). The dashed line indicates chance performance (4\%); Error bars indicate SEM; $(\dagger) P<0.07$.

\section{DISCUSSION}

We trained rats with either complete hippocampal lesions, CA1 lesions, or control surgeries in two different tasks in the watermaze (a conventional watermaze task and delayed match-to-position, DMP). In one condition, rats were trained in the DMP task first, followed by the watermaze task. In another condition, rats were trained in the watermaze task first, followed by the DMP task. Ordinarily, hippocampal lesions and CA1 lesions impair performance in both tasks. Yet, with CA1 lesions, rats were intact in the watermaze task when they had prior DMP training, and they were intact in the DMP task when they had prior watermaze training. In contrast, rats with hippocampal lesions were impaired in both tasks regardless of prior training.

It is perhaps not surprising that rats with large hippocampal lesions did not benefit from prior experience. In earlier work, rats with hippocampal lesions did not benefit from prior training, even when they had been trained on the same kind of task (Bannerman et al. 1995; Moser and Moser 1998; Steele and Morris 1999). In contrast, in our study, rats with CA1 lesions exhibited striking benefits from prior training, even when the prior training involved different tasks. Thus, rats with CA1 lesions acquired the watermaze (after prior DMP training) as well as controls. And they acquired the DMP task (after prior watermaze training) as well as controls. To our knowledge, such a substantial benefit of prior training on the learning of a different task has not been demonstrated in rats with lesions in the hippocampus.

We considered, in the case of the watermaze, that performance might have benefited from the extended interval between surgery and watermaze training ( $43 \mathrm{~d})$ that was needed in order to interpose DMP training. However, watermaze acquisition was not improved in rats with either CA1 or hippocampal lesions when rats were given the same extended surgery-watermaze interval but without prior DMP training (Fig. 5).

We also noted that rats altered their swim pattern as they performed, which might have helped them learn a second task in the watermaze environment. For example, when DMP training was scheduled first, all three groups subsequently spent less time swimming along the edges of the pool during watermaze training than they did when watermaze training was scheduled first (see heat maps in Fig. 3). This effect could have contributed to the finding 
that all three groups performed at least marginally better on the watermaze task if watermaze training was preceded by DMP training (cf. Fig. 3, left and right). However, after DMP training, rats with large hippocampal lesions were still severely impaired at the watermaze in comparison to the other two groups, even with the potential benefit of this swimming strategy. Accordingly, the development of a swim strategy does not readily account for the advantage of prior training found selectively in animals with CA1 lesions.

Why was there a difference between the effects of hippocampal and CA1 lesions when both lesions would be expected to disrupt hippocampal function? One possibility is that large hippocampal lesions caused remote effects, such as volume loss in the cortex (Jarrard and Meldrum 1993; Anagnostaras et al. 2001). Because CA1 lesions spared the majority of the projections to the hippocampus, such lesions could have reduced remote effects (such as volume loss) and thereby preserved function in adjacent structures important for learning. We evaluated the possible role of cortical volume loss in a sample of $60 \%$ of all animals and found a similar, modest reduction in cortical volume after hippocampal and CA1 lesions (8.8\% and $12.7 \%$, respectively). Thus, cortical volume loss does not appear to account for the different findings after hippocampal and CA1 lesions, though we cannot rule out the possible importance of other remote effects caused by hippocampal lesions.

Another possible explanation for the behavioral differences between the two lesion groups turns on the descending projection from area CA3 to the septum (Witter 2007), which remains intact after CA1 lesions but not after large hippocampal lesions. The septum projects to the thalamus (Swanson and Cowan 1979) and might thereby provide an alternative pathway for hippocampal output to reach neocortex. Still, another possibility is that spared CA1 tissue was able to support hippocampal output to neocortex. CA1 lesions did leave nearly $24 \%$ of area CA1 intact, somewhat more than the $14 \%$ that was spared with large hippocampal lesions.

One puzzling aspect of these findings is that DMP performance was intact after CA1 lesions, even without prior experience, once $1 \mathrm{~d}$ of training (four trials) had been given (Fig. 4B,C, left). This finding might reflect spared function in area CA3. Area CA3 has been proposed to be important for one-trial learning (Rolls 2013).

Note that rats with CA1 lesions were impaired at watermaze acquisition and at the early stage of DMP training when they had no prior training but were intact when they had prior training. Accordingly, we suggest that prior experience is the critical factor in understanding the effect of CA1 lesions. As rats gained experience in one of our tasks, conventional watermaze or DMP, they formed memories of many features common to both tasks: a platform is to be found, the platform is not at the edges, distal cues are important. These memories may have gradually organized into a coherent framework, a schema (Morris 2006), which facilitated the learning of new, but related information (see Inglis et al. 2013). Thus, when rats were given DMP training (or watermaze training), they developed a schema from their experience that subsequently facilitated learning in the other task.

Similarly, Tse et al. (2007) demonstrated that schemas can facilitate learning in a flavor-place, paired associate task. After extensive training, rats were able to learn new flavor-place pairs faster than when they had less experience in the task. The current study builds on these findings by demonstrating that schemas can be useful even when a previously learned task and a new task are quite different. Thus, we suggest that experience with the training environment and with features common to the two tasks can be sufficient to form a useful schema. In earlier work with rats, impairment in a visual discrimination task following visual cortical lesions was reduced by prior experience with a conceptually similar task trained in a different modality (Clark and Delay 1991).

Additionally, Tse et al. (2007) demonstrated that once a schema was in place, memories for new flavor-place pairs became rapidly independent of the hippocampus and could survive a hippocampal lesion given as soon as $48 \mathrm{~h}$ after learning. In contrast, hippocampal lesions impaired performance of the paired associate task if lesions were made prior to training (and schema formation). Thus, Tse et al. (2007) suggest that the hippocampus is needed to form schemas, most likely in the neocortex. Note that, in the current study, rats with CA1 lesions exhibited schema-based learning despite receiving lesions prior to training. Because prior experience benefited rats with CA1 lesions, but not rats with large hippocampal lesions, we suggest that hippocampal function (in areas upstream of CA1) may be important for schema formation.

In summary, rats with large hippocampal lesions or restricted CA1 lesions were impaired in the watermaze task and in the DMP task. However, when given prior training with one task, CA1 lesions had no effect on performance in the other task. In contrast, rats with hippocampal lesions did not benefit from prior training. The concept of schema may be useful for understanding the benefits of past experience. Because experience with one task can benefit subsequent learning in a different task, we suggest that features common to the two tasks are required to form a functional schema. This idea leads to the prediction that benefits of prior experience should not be expected across two tasks that are fundamentally different (see Wiltgen et al. 2011).

\section{Materials and Methods}

\section{Subjects}

Subjects were 63 experimentally naive, male Long-Evans rats that received either hippocampal lesions, CA1 lesions, or sham surgeries. After recovery, rats were trained in two spatial tasks: the watermaze task and the DMP task, a variation of the watermaze task that involves one-trial learning. In one condition, training occurred first in the DMP task, followed $2 \mathrm{~d}$ later by the watermaze task (DMP First; $\mathrm{H} n=8$, CA1 $n=8$, sham $n=7$ ). In a second condition, training occurred first in the watermaze task, followed $2 \mathrm{~d}$ later by the DMP task (WM First; $\mathrm{H} n=8$, CA1 $n=8$, sham $n=8$ ). Timelines are shown in Figure 1. Additionally, in a third condition, training in the watermaze task occurred at the same interval after surgery as the DMP First group (43 d), but without prior DMP training (WM After Delay; $\mathrm{H} n=8$, CA1 $n=8$ ). The timeline for this condition is shown in Figure 5. All rats weighed between 320 and 350 $\mathrm{g}$ at the time of surgery. They were maintained on a $12: 12 \mathrm{~h}$ light:dark cycle and were housed individually. Food and water were freely available. All experimental procedures were approved by the Institutional Animal Care and Use Committee at the University of California, San Diego.

\section{Surgery}

Anesthesia was maintained throughout surgery with isoflurane gas $\left(0.8 \%-2.0 \%\right.$ isoflurane delivered in $\mathrm{O}_{2}$ at $\left.1 \mathrm{~L} / \mathrm{min}\right)$. The rat was placed in a Kopf stereotaxic instrument, and the incisor bar was adjusted until Bregma was level with Lambda. For hippocampal and CA1 lesions, ibotenic acid (IBO; Biosearch Technologies) dissolved in $0.1 \mathrm{M}$ PBS (concentration: $10 \mathrm{mg} / \mathrm{mL}, \mathrm{pH} 7.4$ ) was injected into dorsal and ventral regions of the hippocampus using a $10 \mathrm{~mL}, 30-\mathrm{g}$ Hamilton syringe. The syringe was held in a Kopf microinjector (model 5000) and mounted on a stereotaxic frame. The syringe was first lowered to the target coordinate and left in place for 1 min. After injection (at a rate of $0.1 \mathrm{~mL} / \mathrm{min}$ ), the syringe stayed at the target coordinate for 2 min to prevent IBO from spreading up the syringe tract upon its retraction. For CA1 lesions, IBO was injected into 14 sites per hemisphere $(0.025 \mu \mathrm{L} /$ site, unless otherwise noted). For certain injection sites in ventral CA1 (noted below), the syringe was left in place for $5 \mathrm{~min}$ to ensure that IBO 
would not spread up the syringe track (where it might cause unintended damage to CA3 or the dentate gyrus). All coordinates are in millimeters, anteroposterior (AP) relative to Bregma, mediolateral (ML) relative to Lambda, and dorsoventral (DV) relative to the brain surface at $-4.8 \mathrm{~mm}$ from Bregma and $\pm 4.2 \mathrm{~mm}$ from Lambda: $\mathrm{AP}-2, \mathrm{ML} \pm 1, \mathrm{DV}-2.9$; $\mathrm{AP}-3.6, \mathrm{ML} \pm 1, \mathrm{DV}-2.7$; $\mathrm{AP}$ -3.6, ML $\pm 2, \mathrm{DV}-1.9$; $\mathrm{AP}-4.5, \mathrm{ML} \pm 1.4, \mathrm{DV}-3.3$; $\mathrm{AP}-4.5$, ML $\pm 2.7, \mathrm{DV}-1.8$; $\mathrm{AP}-4.5, \mathrm{ML} \pm 4.5, \mathrm{DV}-7.9$ (waited $5 \mathrm{~min}$ before retracting syringe); $\mathrm{AP}-5.3, \mathrm{ML} \pm 3, \mathrm{DV}-1.7 ; \mathrm{AP}-5.3, \mathrm{ML} \pm 4.8, \mathrm{DV}$ -8 (waited 5 min before retracting syringe); $\mathrm{AP}-5.3, \mathrm{ML} \pm 4.8, \mathrm{DV}$ -2.4; $\mathrm{AP}-5.3, \mathrm{ML} \pm 5.8, \mathrm{DV}-7.5 ; \mathrm{AP}-5.3, \mathrm{ML} \pm 5.8, \mathrm{DV}-5.7 ; \mathrm{AP}$ $-5.3, \mathrm{ML} \pm 5.8, \mathrm{DV}-3.9 ; \mathrm{AP}-6.3, \mathrm{ML} \pm 5.4, \mathrm{DV}-3$ (injected 0.05 $\mu \mathrm{L}$ IBO); AP -6.3, ML $\pm 6.3, \mathrm{DV}-5.7$ (injected $0.05 \mu \mathrm{L}$ IBO). For hippocampal lesions, IBO was injected into 18 sites per hemisphere as in Clark et al. (2000). For sham surgeries, rats underwent the same surgical procedures up to the point of the craniotomy. Once awake and responsive, each rat was returned to its home cage for 13-21 d of recovery.

\section{Apparatus}

DMP and watermaze training were conducted in a pool of water (1.8-m diameter at the water level) that was rendered opaque by the addition of powdered milk. The testing room contained a number of constant, salient visual cues (posters, objects, and equipment). A video camera mounted on the ceiling directly above the pool was used in conjunction with a video tracking system (San Diego Instruments) to record the swim path of each rat. An Atlantis platform (12.7-cm diameter) was used that could be raised or lowered remotely (Spooner et al. 1994). In the lowered position, the platform was undetectable and unavailable. In the raised position $(1.5 \mathrm{~cm}$ below the surface of the water), the platform remained invisible, but provided a means to escape the water.

\section{Behavioral training}

\section{DMP}

Rats learned a new platform location each day during three phases of training. Briefly, the platform was moved to a new location at the beginning of each training day, and in the first trial rats had to find the platform without any knowledge of its location. In subsequent trials on the same day, rats could find the platform by recalling where it was located in trial 1.

The first phase consisted of five training days. Each day, a trial began when a rat was placed in the water facing the pool wall at one of four start points (which were changed for each trial and counterbalanced across animals during training). The platform was kept in the raised position throughout the trial, allowing the rats to escape from the water. Rats were given up to 2 min to escape the water before being guided to the platform by the experimenter. After escaping the water, rats remained on the platform for $30 \mathrm{sec}$ before being returned to their home cage. Three more training trials were given on the same day using the same platform location (15-sec delay between trials). Performance was measured as the distance traveled to reach the platform, averaged across trials $2-4$ each day.

The second phase consisted of 12 training days. Each day, rats received two training trials with either a 1-min, a 20-min, or a 6-h delay between the two trials. Rats were tested with each of the three delays on four separate days. The order in which the delays were given was mixed and counterbalanced across animals. Performance with each delay was measured as the distance traveled to reach the platform during trial 2 , averaged across the four times the rats experienced that delay.

The third phase of the DMP task was conducted in $1 \mathrm{~d}$. Rats received one training trial with a new platform location and one reinforced probe trial separated by a 1-min delay. The probe trials were similar to the training trials, except that the platform was lowered for the first $60 \mathrm{sec}$. After the platform was raised, rats were given up to $1 \mathrm{~min}$ to escape the water before being guided to the platform by the experimenter. Rats remained on the platform for $30 \mathrm{sec}$ before being returned to their home cage. Performance was measured as the percentage of time that each rat spent in the circular zone directly above the platform location during the first 60 sec of the probe trial (chance $=4 \%$ ).

\section{Watermaze acquisition}

Rats learned a single platform location across $5 \mathrm{~d}$ of training. There were 5 trials/day: a reinforced probe trial followed by four training trials (as described above for DMP training) with delays between the trials of about $10 \mathrm{~min}$. As with the DMP task, the start point was changed for each trial and counterbalanced across animals during training. Performance was measured as the percentage of time that each rat spent in the circular zone directly above the platform location during the first $60 \mathrm{sec}$ of the probe trial at the beginning of each day $($ chance $=4 \%)$.

\section{Histology}

At completion of testing, the rats were administered an overdose of sodium pentobarbital and perfused transcardially with buffered $0.9 \% \mathrm{NaCl}$ solution followed by $10 \%$ formaldehyde solution (in $0.1 \mathrm{M}$ phosphate buffer). The brains were removed and cryoprotected in $20 \%$ glycerol/10\% formaldehyde. Coronal sections (50 $\mu \mathrm{m})$ were cut with a freezing microtome beginning at the level of the anterior commissure and continuing caudally through the length of the hippocampus. Every fifth section was mounted and stained with thionin to assess the extent of the lesions.

Images of the thionin-stained tissue sections were acquired using a DM6000 microscope (Leica Microsystems, Inc.). The images from every other mounted section were then analyzed using Stereo Investigator software (mbf Bioscience; MicroBrightField). The volumes of spared tissue in CA1, CA3, and DG were calculated using the Cavalieri method (as in Hales et al. 2014). This analysis was conducted for all lesion rats and eight sham rats (four from the WM First condition, four from the DMP First condition).

\section{Acknowledgments}

We thank J. Hales, L. Johnson, M. Sapiurka, A. Cawley-Bennett, A. Hashi, M. Gosselin, M. Graves, S. Vincent, and Y. Arellano for assistance. This work was supported by the Medical Research Service of the Department of Veterans Affairs (L.S. 101 CX000359; R.C. I01BX001076), NSF grant (\#SMA-1041755) to the Temporal Dynamics of Learning Center at UCSD, and NIMH (MH24600).

\section{REFERENCES}

Anagnostaras SG, Gale GD, Fanselow MS. 2001. Hippocampus and contextual fear conditioning: recent controversies and advances. Hippocampus 11: 8-17.

Bannerman DM, Good MA, Butcher SP, Ramsay M, Morris RGM. 1995. Distinct components of spatial learning revealed by prior training and NMDA receptor blockade. Nature 378: 182-186.

Bartlett FC. 1932. Remembering: a study in experimental and social psychology. Cambridge, England: Cambridge University Press.

Bransford JD, Johnson MK. 1972. Contextual prerequisites for understanding: some investigations of comprehension and recall. J Verbal Learning Verbal Behav 11: 717-726.

Clark RE, Delay ER. 1991. Reduction of lesion-induced deficits in visual reversal learning following cross-modal training. Restorative Neurol Neurosci 3: 247-255.

Clark RE, Zola SM, Squire LR. 2000. Impaired recognition memory in rats after damage to the hippocampus. J Neurosci 20: 8853-8860.

Dragoi G, Tonegawa S. 2013. Development of schemas revealed by prior experience and NMDA receptor knock-out. Elife 2: e01326.

Hales JB, Schlesiger MI, Leutgeb JK, Squire LR, Leutgeb S, Clark RE. 2014. Medial entorhinal cortex lesions only partially disrupt hippocampal place cells and hippocampus-dependent place memory. Cell Rep 9: 893-901.

Inglis J, Martin SJ, Morris RG. 2013. Upstairs/downstairs revisited: spatial pretraining-induced rescue of normal spatial learning during selective blockade of hippocampal N-methyl-d-aspartate receptors. Eur J Neurosci 37: 718-727.

Jarrard LE, Meldrum BS. 1993. Selective excitotoxic pathology in the rat hippocampus. Neuropathol Appl Neurobiol 19: 381-389. 
Maguire EA, Frith CD, Morris RG. 1999. The functional neuroanatomy of comprehension and memory: the importance of prior knowledge. Brain 122: $1839-1850$.

McKenzie S, Frank AJ, Kinsky NR, Porter B, Rivière PD, Eichenbaum H. 2014. Hippocampal representation of related and opposing memories develop within distinct, hierarchically-organized neural schemas. Neuron 83: 202-215.

Morris RG. 2006. Elements of a neurobiological theory of hippocampal function: the role of synaptic plasticity, synaptic tagging and schemas. Eur J Neurosci 11: 2829-2846.

Moser MB, Moser EI. 1998. Distributed encoding and retrieval of spatial memory in the hippocampus. J Neurosci 18: 7535-7542.

Ocampo AC, Squire LR, Clark RE. 2017. Hippocampal area CA1 and remote memory in rats. Learn Mem. 24: 563-568.

Otnaess MK, Brun VH, Moser MB, Moser EI. 1999. Pretraining prevents spatial learning impairment after saturation of hippocampal long-term potentiation. J Neurosci 19: RC49.

Race E, Palombo DJ, Cadden M, Burke K, Verfaellie M. 2015. Memory integration in amnesia: prior knowledge supports verbal short-term memory. Neuropsychologia 70: 272-280.

Rolls ET. 2013. A quantitative theory of the functions of the hippocampal CA3 network in memory. Front Cell Neurosci 7: 98.

Spooner RI, Thomson A, Hall J, Morris RG, Salter SH. 1994. The Atlantis platform: a new design and further developments of
Buresova's on-demand platform for the water maze. Learn Mem 1: 203-211.

Steele RJ, Morris RG. 1999. Delay-dependent impairment of a matching-toplace task with chronic and intrahippocampal infusion of the NMDAantagonist D-AP5. Hippocampus 9: 118-136.

Swanson LW, Cowan WM. 1979. The connections of the septal region in the rat. J Comp Neurol 186: 621-655.

Tse D, Langston RF, Kakeyama M, Bethus I, Spooner PA, Wood ER, Witter MP, Morris RG. 2007. Schemas and memory consolidation. Science 316: 76-82.

van Kesteren MT, Beul SF, Takashima A, Henson RN, Ruiter DJ, Fernández G. 2013. Differential roles for medial prefrontal and medial temporal cortices in schema-dependent encoding: from congruent to incongruent. Neuropsychologia 51: 2352-2359.

van Strien NM, Cappaert NLM, Witter MP. 2009. The anatomy of memory: an interactive overview of the parahippocampal-hippocampal network. Nat Rev Neurosci 10: 272-282.

Wiltgen BJ, Wood AN, Levy B. 2011. The cellular mechanisms of memory are modified by experience. Learn Mem 18: 747-750.

Witter MP. 2007. Intrinsic and extrinsic wiring of CA3: indications for connectional heterogeneity. Learn Mem 14: 705-713.

Received September 15, 2017; accepted in revised form December 8, 2017. 


\section{Corrigendum}

Learning \& Memory 25: 115-121 (2018)

Corrigendum: The beneficial effect of prior experience on the acquisition of spatial memory in rats with CA1, but not large hippocampal lesions: a possible role for schema formation

Amber C. Ocampo, Larry R. Squire, and Robert E. Clark

In the above-mentioned article, the citation at the top of page 117, column 2, to "Fig. 3A, right" should instead call out Fig. 4B, right. This has already been corrected online.

The author apologizes for this error.

In addition, a production error occurred that resulted in the inadvertent duplication of two passages, both of which have been corrected online.

In the PDF, page 117, top paragraph in second column, two sentences were erroneously inserted: "However, in contrast to the findings..." and "The H group was impaired relative to the other two groups..."

In the PDF, pages 117 and 118, beginning at the bottom of the right column on page 117, four sentences were erroneously inserted: "In the DMP First condition, mean distance swum..." and "A repeated-measures ANOVA demonstrated differences in performance..." and "Post hoc, pairwise comparisons showed that the CA1 group performed..." and "The results were the same in the WM First condition..."

The publisher apologizes for any confusion this may have caused.

doi: $10.1101 / \mathrm{lm} .047472 .118$ 


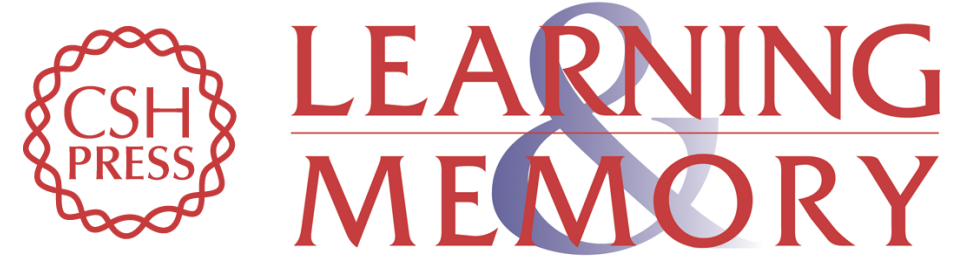

\title{
The beneficial effect of prior experience on the acquisition of spatial memory in rats with CA1, but not large hippocampal lesions: a possible role for schema formation
}

Amber C. Ocampo, Larry R. Squire and Robert E. Clark

Learn. Mem. 2018, 25:

Access the most recent version at doi:10.1101//m.046482.117

\begin{abstract}
Related Content Corrigendum: The beneficial effect of prior experience on the acquisition of spatial memory in rats with CA1, but not large hippocampal lesions: a possible role for schema formation

Amber C. Ocampo, Larry R. Squire and Robert E. Clark

Learn. Mem. April , 2018 25: 197

References This article cites 25 articles, 8 of which can be accessed free at:

http://learnmem.cshlp.org/content/25/3/115.full.html\#ref-list-1

Articles cited in:

http://learnmem.cshlp.org/content/25/3/115.full.html\#related-urls

Creative This article is distributed exclusively by Cold Spring Harbor Laboratory Press for the Commons first 12 months after the full-issue publication date (see

License http://learnmem.cshlp.org/site/misc/terms.xhtml). After 12 months, it is available under a Creative Commons License (Attribution-NonCommercial 4.0 International), as described at http://creativecommons.org/licenses/by-nc/4.0/.

Email Alerting Receive free email alerts when new articles cite this article - sign up in the box at the Service top right corner of the article or click here.
\end{abstract}

